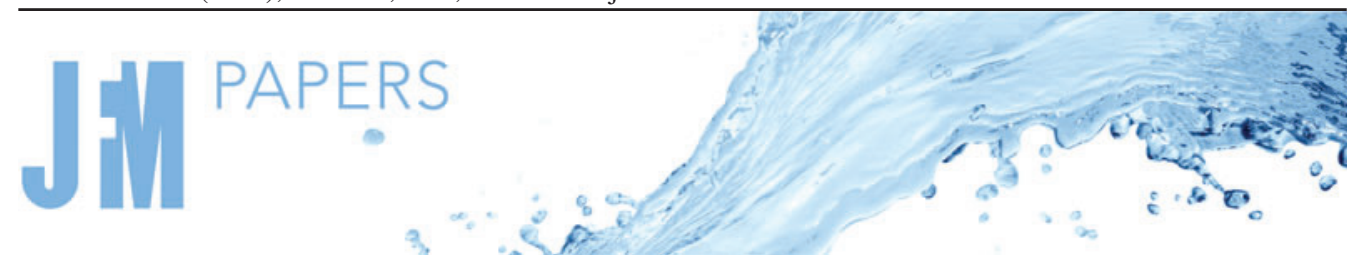

\title{
One-point statistics for turbulent pipe flow up to $R e_{\tau} \approx 6000$
}

\author{
Sergio Pirozzoli ${ }^{1} \uparrow$, Joshua Romero ${ }^{2}$, Massimiliano Fatica $^{2}$, \\ Roberto Verzicco ${ }^{3,4}$ and Paolo Orlandi ${ }^{1}$ \\ ${ }^{1}$ Dipartimento di Ingegneria Meccanica e Aerospaziale, Sapienza Università di Roma, Via Eudossiana 18, \\ 00184 Roma, Italy \\ ${ }^{2}$ NVIDIA Corporation, 2701 San Tomas Expressway, Santa Clara, CA 95050, USA \\ ${ }^{3}$ Dipartimento di Ingegneria Industriale, Università di Roma TorVergata, Via del Politecnico 1, 00133 \\ Roma, Italy \\ ${ }^{4}$ Physics of Fluid Group, University of Twente, PO Box 217, 7500 AE Enschede, The Netherlands
}

(Received 23 March 2021; revised 5 August 2021; accepted 12 August 2021)

We study turbulent flows in a smooth straight pipe of circular cross-section up to friction Reynolds number $\left(R e_{\tau}\right) \approx 6000$ using direct numerical simulation (DNS) of the Navier-Stokes equations. The DNS results highlight systematic deviations from Prandtl friction law, amounting to approximately $2 \%$, which would extrapolate to approximately $4 \%$ at extreme Reynolds numbers. Data fitting of the DNS friction coefficient yields an estimated von Kármán constant $k \approx 0.387$, which nicely fits the mean velocity profile, and which supports universality of canonical wall-bounded flows. The same constant also applies to the pipe centreline velocity, thus providing support for the claim that the asymptotic state of pipe flow at extreme Reynolds numbers should be plug flow. At the Reynolds numbers under scrutiny, no evidence for saturation of the logarithmic growth of the inner peak of the axial velocity variance is found. Although no outer peak of the velocity variance directly emerges in our DNS, we provide strong evidence that it should appear at $R e_{\tau} \gtrsim 10^{4}$, as a result of turbulence production exceeding dissipation over a large part of the outer wall layer, thus invalidating the classical equilibrium hypothesis.

Key words: pipe flow, turbulence simulation, turbulence theory

\section{Introduction}

Turbulent flow in circular pipes has always attracted the interest of scientists, owing to its prominent importance in the engineering practice and because of the beautiful simplicity of the set-up. In this respect, the pioneering flow visualizations of Reynolds

$\dagger$ Email address for correspondence: sergio.pirozzoli@uniroma1.it

(C) The Author(s), 2021. Published by Cambridge University Press. This is an Open Access article, distributed under the terms of the Creative Commons Attribution licence (https://creativecommons. org/licenses/by/4.0/), which permits unrestricted re-use, distribution, and reproduction in any medium, provided the original work is properly cited. 


\section{S. Pirozzoli and others}

(1883) may be regarded as a milestone for the understanding of turbulent and transitional flows. The most extensive experimental measurements of high-Reynolds-number pipe flows have been carried out in modern times in the Princeton SuperPipe pressurized facility (Zagarola \& Smits 1998; McKeon, Zagarola \& Smits 2005; Hultmark, Bailey \& Smits 2010). Those investigations have allowed scientists to measure the main flow features such as friction and mean velocity profiles with high precision, and they currently constitute the most comprehensive database for the study of pipe turbulence. However, even the use of specialized microfabricated hot-wire probes could not provide fully reliable information about the viscous and buffer layers at high Reynolds numbers (Hultmark et al. 2012). Additional experimental studies of pipe turbulence have been carried out in the high-Reynolds-number actual flow facility (Hi-Reff), a water tunnel with relatively large diameter, which allows for accurate estimation of friction (Furuichi et al. 2015, 2018). Recently, the Center for International Cooperation in Long Pipe Experiments (CICLoPE) facility of the University of Bologna (Fiorini 2017; Willert et al. 2017) has been set up, whose large diameter (approximately $1 \mathrm{~m}$ ) offers a well-established turbulent flow with relatively large viscous scales, thus granting higher spatial resolution. Flows in different facilities seem to have sensibly different properties in terms of friction and mean velocity profiles, which we will comment on.

Numerical simulation of pipe turbulence flow has received less interest than other canonical flows, the plane channel in particular, because of additional difficulties involved with the discrete solution of the Navier-Stokes equations in cylindrical coordinates, with special reference to the treatment of the geometrical singularity at the pipe axis. Early numerical simulations of turbulent pipe flow were carried out by Eggels et al. (1994), at friction Reynolds number $R e_{\tau}=180\left(\operatorname{Re}_{\tau}=u_{\tau} R / \nu\right.$, with $u_{\tau}=\left(\tau_{w} / \rho\right)^{1 / 2}$ the friction velocity, $R$ the pipe radius and $v$ the fluid kinematic viscosity). Effects of drag reduction associated with pipe rotation were later studied by Orlandi \& Fatica (1997). Higher Reynolds numbers (up to $R e_{\tau} \approx 1140$ ) were reached by Wu \& Moin (2008), which first allowed one to observe a near logarithmic layer in the mean velocity profile. Flow visualizations and two-point correlation statistics pointed to the existence of high-speed wavy structures in the pipe core region which are elongated in the axial direction, and whose streamwise and azimuthal dimensions do not change substantially with the Reynolds number, when normalized in outer units. Further follow-up direct numerical simulation (DNS) studies have been carried out by El Khoury et al. (2013), Chin et al. (2014) and Ahn et al. (2013). At present, the highest Reynolds number in pipe flow $\left(R e_{\tau} \approx 3000\right)$ has been reached in the study of Ahn et al. (2015). Although no sizeable logarithmic layer is present yet at those conditions, some effects associated with significant scale separation between inner- and outer-scale turbulence were observed, as the presence of a $k^{-1}$ ( $k$ being the wavenumber in any wall-parallel direction) power-law ranges in the velocity spectra.

Despite inherent limitations in the Reynolds numbers which can be attained, DNS has the advantage over experiments of yielding immediate access to the near-wall region, and of allowing scientists to measure some flow properties, e.g. the turbulence dissipation rate, which can hardly be measured in experiments. Hence, it is generally claimed that DNS data at increasing Reynolds numbers are needed to prove or disprove theoretical claims related to departure (or not) of the statistical properties of wall-bounded turbulence from the universal wall scaling (Cantwell 2019; Chen \& Sreenivasan 2021; Monkewitz 2021). In this paper we thus present DNS data of turbulent flow in a smooth circular pipe at $R e_{\tau} \approx 6000$, which is two times higher than the previous state of the art. Relying on the DNS data, we revisit current theoretical inferences and discuss implications about possible trends in the extreme Reynolds number regime. 


\section{The numerical dataset}

The code used for DNS is the spin-off of an existing solver previously used to study Rayleigh-Bénard convection in cylindrical containers at extreme Rayleigh numbers (Stevens et al. 2013). That code is in turn the evolution of the solver originally developed by Verzicco \& Orlandi (1996), and used for DNS of pipe flow by Orlandi \& Fatica (1997). A second-order finite-difference discretization of the incompressible Navier-Stokes equations in cylindrical coordinates is used, based on the classical marker-and-cell method (Harlow \& Welch 1965), with staggered arrangement of the flow variables to remove odd-even decoupling phenomena and guarantee discrete conservation of the total kinetic energy in the inviscid flow limit. Uniform volumetric forcing is applied to the axial momentum equation to maintain constant mass flow rate in time. The Poisson equation resulting from enforcement of the divergence-free condition is efficiently solved by double trigonometric expansion in the periodic axial and azimuthal directions, and inversion of tridiagonal matrices in the radial direction (Kim \& Moin 1985). An extensive series of previous studies about wall-bounded flows from this group proved that second-order finite-difference discretization yields in practical cases of wall-bounded turbulence results which are by no means inferior in quality to those of pseudospectral methods (e.g. Moin \& Verzicco 2016; Pirozzoli, Bernardini \& Orlandi 2016). A crucial issue is the proper treatment of the polar singularity at the pipe axis. A detailed description of the subject is reported in Verzicco \& Orlandi (1996), but basically, the radial velocity $u_{r}$ in the governing equations is replaced by $q_{r}=r u_{r}$ ( $r$ is the radial space coordinate), which by construction vanishes at the axis. The governing equations are advanced in time by means of a hybrid third-order low-storage Runge-Kutta algorithm, whereby the diffusive terms are handled implicitly, and convective terms in the axial and radial direction are handled explicitly. An important issue in this respect is the convective time step limitation in the azimuthal direction, due to intrinsic shrinking of the cells' size toward the pipe axis. To alleviate this limitation we rely on implicit treatment of the convective terms in the azimuthal direction (Akselvoll \& Moin 1996; Wu \& Moin 2008), which enables marching in time with similar time step as in planar domains flow in practical computations. In order to minimize numerical errors associated with implicit time stepping, in the present code explicit and explicit discretizations of the azimuthal convective terms are linearly blended with the radial coordinate, in such a way that near the pipe wall the treatment is fully explicit, and near the pipe axis it is fully implicit. The code was adapted to run on clusters of graphic accelerators (GPUs), using a combination of CUDA Fortran and OpenACC directives, and relying on the CUFFT libraries for efficient execution of fast Fourier transforms (FFTs) (Ruetsch \& Fatica 2014). The DNS were carried out on the Marconi-100 machine based at CINECA (Italy), relying on NVIDIA Volta V100 graphic cards. Specifically, 1024 GPUs were used for DNS-F.

Numerical simulations are carried out with periodic boundary conditions in the axial $(z)$ and azimuthal $(\theta)$ directions. The velocity field is then controlled by two parameters, namely the bulk Reynolds number $\left(R e_{b}=2 R u_{b} / v\right.$, with $R$ the pipe radius, $u_{b}$ the fluid bulk velocity and $v$ its kinematic viscosity), and the relative pipe length, $L_{z} / R$. A list of the main simulations that we have carried out is given in table 1 . The mesh resolution is designed based on well-established criteria in the wall turbulence community. In particular, the collocation points are distributed in the wall-normal direction so that approximately 30 points are placed within $y^{+} \leq 40(y=R-r$ is the wall distance, and the + superscript is used to denote normalization with respect to $u_{\tau}$ and $v$ ), with the first grid point at $y^{+} \approx 0.05$. The mesh is progressively stretched in the outer wall layer in such a way that the mesh spacing is proportional to the local Kolmogorov length 


\begin{tabular}{|c|c|c|c|c|c|c|c|}
\hline Dataset & $L_{z} / R$ & $\operatorname{Mesh}\left(N_{\theta} \times N_{r} \times N_{z}\right)$ & $\operatorname{Re}_{b}$ & $\lambda$ & $R e_{\tau}$ & $T / \tau_{t}$ & Line style \\
\hline DNS-A & 15 & $256 \times 67 \times 256$ & 5300 & 0.03700 & 180.3 & 204.0 & \\
\hline DNS-B & 15 & $768 \times 140 \times 768$ & 17000 & 0.02716 & 495.3 & 87.4 & \\
\hline DNS-C & 15 & $1792 \times 270 \times 1792$ & 44000 & 0.02136 & 1136.6 & 25.9 & \\
\hline DNS-C-SH & 7.5 & $1792 \times 270 \times 986$ & 44000 & 0.02164 & 1144.2 & 31.1 & NA \\
\hline DNS-C-LO & 30 & $1792 \times 270 \times 3944$ & 44000 & 0.02128 & 1134.6 & 24.5 & NA \\
\hline DNS-C-FT & 15 & $3944 \times 270 \times 1792$ & 44000 & 0.02114 & 1131.0 & 31.3 & NA \\
\hline DNS-C-FR & 15 & $1792 \times 540 \times 1792$ & 44000 & 0.02132 & 1135.7 & 28.6 & NA \\
\hline DNS-C-FZ & 15 & $1792 \times 270 \times 3944$ & 44000 & 0.02132 & 1135.7 & 15.5 & NA \\
\hline DNS-D & 15 & $3072 \times 399 \times 3072$ & 82500 & 0.01836 & 1976.0 & 22.4 & \\
\hline DNS-E & 15 & $4608 \times 540 \times 4608$ & 133000 & 0.01659 & 3028.1 & 16.6 & \\
\hline DNS-F & 15 & $9216 \times 910 \times 9216$ & 285000 & 0.01428 & 6019.4 & 8.32 & \\
\hline
\end{tabular}

Table 1. Flow parameters for DNS of pipe flow. Here $R$ is the pipe radius; $L_{z}$ is the pipe axial length; $N_{\theta}, N_{r}$ and $N_{z}$ are the number of grid points in the azimuthal, radial and axial directions, respectively; $R e_{b}=2 R u_{b} / v$ is the bulk Reynolds number; $\lambda=8 \tau_{w} /\left(\rho u_{b}^{2}\right)$ is the friction factor; $R e_{\tau}=u_{\tau} R / v$ is the friction Reynolds number; $T$ is the time interval used to collect the flow statistics; and $\tau_{t}=R / u_{\tau}$ is the eddy turnover time.

scale, which there varies as $\eta^{+} \approx 0.8 y^{+1 / 4}$ (Jiménez 2018), and the radial spacing at the pipe axis is $\Delta y^{+} \approx 8.8$. Additional details are provided in a specifically focused publication (Pirozzoli \& Orlandi 2021). Regarding the axial and azimuthal directions, finite-difference simulations of wall-bounded flows yield grid-independent results as long as $\Delta x^{+} \approx 10, R^{+} \Delta \theta \approx 4.5$ (Pirozzoli et al. 2016), hence the associated number of grid points scales as $N_{z} \approx L_{z} / R \times R e_{\tau} / 10, N_{\theta} \sim 2 \pi \times R e_{\tau} / 4.5$. All DNS have been carried out at Courant-Friedrichs-Lewy (CFL) number close to unity, based on the radial convective time step limitation. The CFL number along the axial direction is typically smaller by a factor two. The time step expressed in wall units $\left(v / u_{\tau}^{2}\right)$ ranges from $\Delta t^{+}=0.55$ in DNS-A to $\Delta t^{+}=0.15$ in DNS-F. According to the established practice (Hoyas \& Jiménez 2006; Ahn et al. 2015; Lee \& Moser 2015), the time intervals used to collect the flow statistics are reported in terms of eddy-turnover times, $\tau_{t}=R / u_{\tau}$. For reference, the time window used to collect the flow statistics in DNS-F amounts to approximately 13.1 flow-through times $\left(L_{z} / u_{b}\right.$ time units).

The sampling errors for some key properties discussed in this paper have been estimated using the method of Russo \& Luchini (2017), based on an extension of the classical batch means approach. The results of the uncertainty estimation analysis are listed in table 2, where we provide expected values and associated standard deviation for the friction factor $(f)$, mean centreline velocity $\left(U_{C L}\right)$, peak axial velocity variance and its position $\left(\left(\left\langle u_{z}^{2}\right\rangle_{I P}\right.\right.$ and $y_{I P}$, respectively), and the dissipation rate of axial velocity variance $\left(\epsilon_{11 w}\right)$. Here and elsewhere, capital letters are used to denote flow properties averaged in the homogeneous spatial directions and in time, brackets denote the averaging operator, and lower-case letters to denote fluctuations from the mean. We find that the sampling error is generally quite limited, being larger in the largest DNS, which have been run for shorter times. In particular, in DNS-F the expected sampling error in friction, centreline velocity and peak velocity variance is approximately $0.5 \%$, whereas it is approximately $1 \%$ for the wall dissipation. Additional tests aimed at establishing the effect of axial domain length and grid size have been carried out for the DNS-C flow case, whose results are also reported in table 2 . We find that doubling the pipe length yields a change in the basic flow properties of approximately $0.2 \%-0.3 \%$, whereas halving it yields changes of approximately $1 \%$ in friction and peak velocity variance, and up to $10 \%$ in the wall 


$\begin{array}{lccccc}\text { Dataset } & \lambda & U_{C L}^{+} & \left\langle u_{z}^{2}\right\rangle_{I P}^{+} & y_{I P}^{+} & \epsilon_{11 w}^{+} \\ \text {DNS-A } & 0.03700 \pm 0.15 \% & 19.30 \pm 0.087 \% & 7.129 \pm 0.26 \% & 14.95 \pm 0.24 \% & 0.1168 \pm 0.47 \% \\ \text { DNS-B } & 0.02716 \pm 0.074 \% & 21.81 \pm 0.17 \% & 7.352 \pm 0.17 \% & 14.28 \pm 0.010 \% & 0.1506 \pm 0.21 \% \\ \text { DNS-C } & 0.02136 \pm 0.13 \% & 24.07 \pm 0.18 \% & 7.995 \pm 0.29 \% & 14.66 \pm 0.073 \% & 0.1697 \pm 0.37 \% \\ \text { DNS-C-SH } & 0.02164 \pm 0.14 \% & 24.09 \pm 0.20 \% & 8.071 \pm 0.44 \% & 14.37 \pm 0.11 \% & 0.1952 \pm 0.54 \% \\ \text { DNS-C-LO } & 0.02128 \pm 0.16 \% & 24.17 \pm 0.11 \% & 7.965 \pm 0.29 \% & 14.62 \pm 0.058 \% & 0.1704 \pm 0.40 \% \\ \text { DNS-C-FT } & 0.02114 \pm 0.12 \% & 24.28 \pm 0.14 \% & 7.948 \pm 0.27 \% & 14.66 \pm 0.078 \% & 0.1691 \pm 0.34 \% \\ \text { DNS-C-FR } & 0.02132 \pm 0.25 \% & 24.10 \pm 0.12 \% & 7.886 \pm 0.31 \% & 14.41 \pm 0.096 \% & 0.1741 \pm 0.60 \% \\ \text { DNS-C-FZ } & 0.02132 \pm 0.21 \% & 24.07 \pm 0.26 \% & 8.168 \pm 0.38 \% & 14.89 \pm 0.14 \% & 0.1727 \pm 0.44 \% \\ \text { DNS-D } & 0.01839 \pm 0.25 \% & 25.56 \pm 0.34 \% & 8.397 \pm 0.43 \% & 14.79 \pm 0.098 \% & 0.1822 \pm 0.57 \% \\ \text { DNS-E } & 0.01658 \pm 0.26 \% & 26.47 \pm 0.27 \% & 8.681 \pm 0.69 \% & 14.87 \pm 0.13 \% & 0.1903 \pm 0.93 \% \\ \text { DNS-F } & 0.01428 \pm 0.36 \% & 28.05 \pm 0.35 \% & 9.108 \pm 0.72 \% & 15.14 \pm 0.20 \% & 0.1993 \pm 1.10 \%\end{array}$

Table 2. Uncertainty estimation study: mean values of representative quantities and standard deviation of their estimates. Here $\lambda$ is the friction factor; $U_{C L}^{+}$is the mean pipe centreline velocity; $\left\langle u_{z}^{2}\right\rangle_{I P}^{+}$is the peak axial velocity variance and $y_{I P}^{+}$is its distance from the wall; and $\epsilon_{11 w}^{+}$is the dissipation rate of $\left\langle u_{z}^{2}\right\rangle$ at the wall.

dissipation. Hence, consistent with previous studies (Chin et al. 2010), we believe that the selected pipe length $\left(L_{z} / R=15\right)$ is representative of an infinitely long pipe, at least for the purposes of the present study. In order to quantify uncertainties associated with numerical discretization, additional simulations have been carried out by doubling the grid points in the azimuthal, radial and axial directions. Based on the data reported in the table, after discarding the short pipe case, we can thus quantify the uncertainty due to numerical discretization and limited pipe length to be approximately $0.3 \%$ for the friction coefficient and pipe centreline velocity, $0.6 \%$ for the peak velocity variance and $0.9 \%$ for the wall dissipation.

\section{Results}

Qualitative information about the structure of the flow field is provided by instantaneous perspective views of the axial velocity field, provided in figure 1. Although finer-scale details are visible at the higher $R e$, the flow in the cross-stream planes is always characterized by a limited number of bulges distributed along the azimuthal direction, which closely recall the proper orthogonal decomposition (POD) modes identified by Hellström \& Smits (2014), and which correspond to alternating intrusions of high-speed fluid from the pipe core and ejections of low-speed fluid from the wall. Streaks are visible in the near-wall cylindrical shells, whose organization has clear association with the cross-stream pattern. Specifically, regardless of the Reynolds number, $R$-sized low-streaks are observed in association with large-scale ejections, whereas $R$-sized high-speed streaks occur in the presence of large-scale inrush from the core flow. At the same time, smaller streaks scaling in wall units appear, corresponding to buffer-layer ejections/sweeps. Hence, organization of the flow on at least two length scales is apparent here, whose separation increases with $R e_{\tau}$.

Mean friction is obviously a parameter of paramount importance as it is related to power expenditure to sustain the flow. In figure 2, we show the friction factor, namely

$$
\lambda=\frac{8 \tau_{w}}{\rho u_{b}^{2}} .
$$




\section{S. Pirozzoli and others}
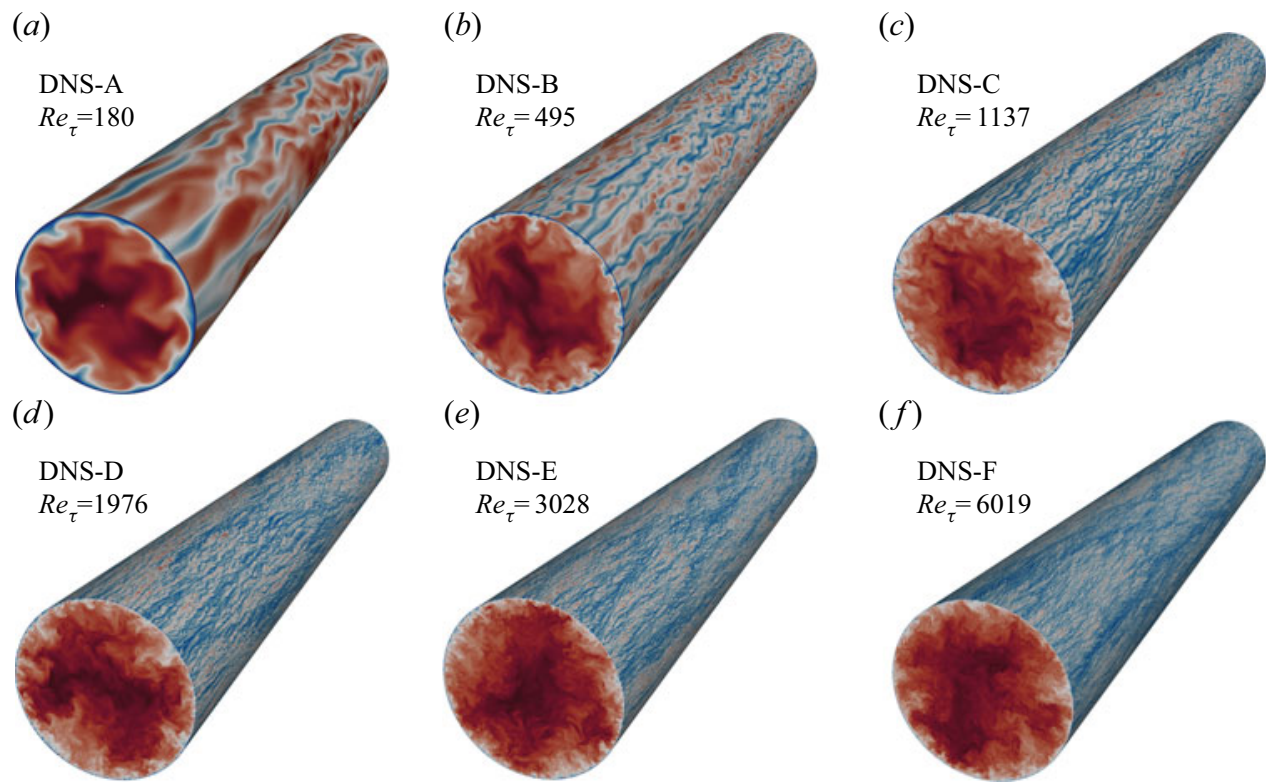

Figure 1. Instantaneous axial velocity contours (colour scale from blue to red) in turbulent pipe flow as obtained from DNS. Contours are shown on a cross-stream plane and on a near-wall cylindrical shell $\left(y^{+} \approx 15\right)$.

A correlation generally used for smooth pipes is the Prandtl friction law,

$$
1 / \lambda^{1 / 2}=A \log _{10}\left(\operatorname{Re}_{b} \lambda^{1 / 2}\right)-B,
$$

where $A=\log 10 /(2 k \sqrt{2})$, with $k$ being the von Kármán log-law constant. The standard values $A=2.0, B=0.8$, were derived by fitting the experimental data of Nikuradse (1933). Reynolds-number-dependent corrections to the standard friction law were introduced by McKeon et al. (2005) in order to improve the fitting of the SuperPipe data. Figure 2 shows overall agreement of all DNS and experimental data with the Prandtl law. However, closer scrutiny (see the figure insets) highlights some scatter. Regarding DNS, all datasets overshoot the Prandtl law at low Reynolds number, although to a quite different extent. In fact, the data of Wu \& Moin (2008), El Khoury et al. (2013) and Chin et al. (2014) exceed the theoretical values by up to $4 \%$, whereas our data tend to be much more consistent with those of Ahn et al. (2015). We believe that this difference may be related to different grid resolution in the azimuthal direction, which was $R^{+} \Delta \theta=7-8$ in those previous studies, and 4-5 in our DNS. Our data in fact show minimal overshoot at low Reynolds number, and consistent undershoot from Prandtl law by approximately $2 \%$. Regarding experiments, SuperPipe data typically tend to lie above the theoretical curve by approximately $2 \%$, whereas the CICLoPE and Hi-Reff data tend to fall short of it. Although the range of data overlap is not extensive, it appears that DNS data tend to be more consistent with the CICLoPE and Hi-Reff data than with other datasets. Fitting the current DNS data with a functional relationship as (3.2), yields $A \approx 2.102, B \approx 1.148$, with an inferred value of the von Kármán constant of $k=0.387 \pm 0.004$, with uncertainty estimates based on $95 \%$ confidence bounds from the curve-fitting procedure. This value is extremely close to that suggested by Furuichi et al. (2018), who reported $k=0.386$ as an average value over a very wide range of Reynolds numbers, and also very close to values reported in boundary layers (Nagib \& Chauhan 2009) and channels (Lee \& Moser 2015). 

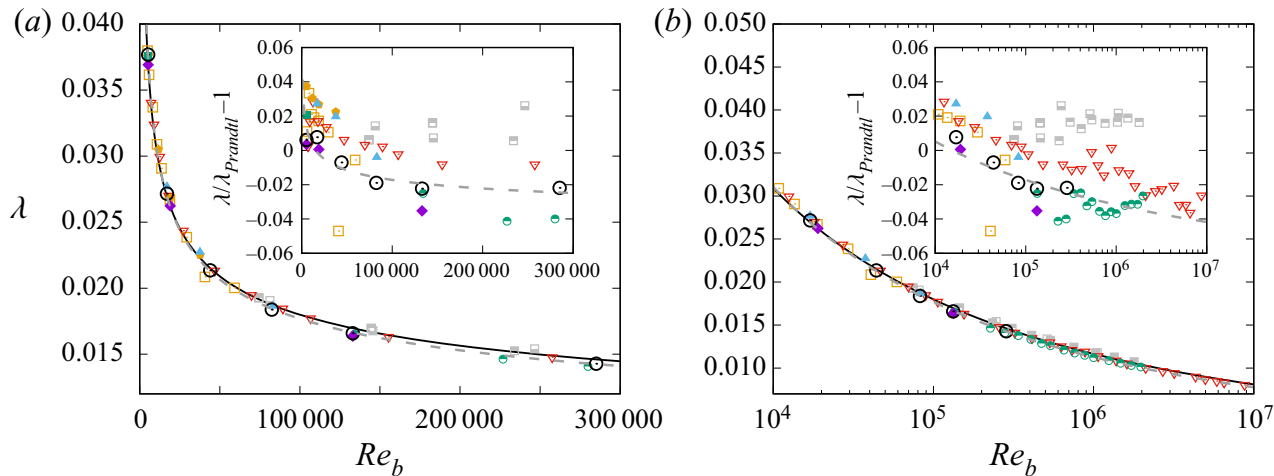

Figure 2. Friction factor as a function of bulk Reynolds number, in linear (a) and in semilogarithmic $(b)$ scale. Circles denote present DNS data, other symbols are defined in table 3. The solid line corresponds to the classical Prandtl friction law as given in (3.2), whereas the dashed grey line corresponds to a fit of the DNS data. Relative deviations with respect to the Prandtl friction law are shown in the insets.

\begin{tabular}{|c|c|c|c|}
\hline Source & Type & $R e_{\tau}$ range & Symbols \\
\hline Wu \& Moin (2008) & DNS & 180,1140 & - \\
\hline El Khoury et al. (2013) & DNS & $180-1000$ & 0 \\
\hline Chin et al. (2014) & DNS & $180-2000$ & $\Delta$ \\
\hline Ahn et al. (2013), Ahn et al. (2015) & DNS & $180-3000$ & $>$ \\
\hline Durst, Jovanović \& Sender (1995) & EXP & 250 & $\diamond$ \\
\hline Swanson et al. (2002) & EXP & 170-1500 & $\square$ \\
\hline Fiorini (2017) & EXP & $3000-35000$ & $\theta$ \\
\hline Willert et al. (2017) & EXP & $5400-40000$ & (1) \\
\hline Nagib et al. (2017) & EXP & $8000-40000$ & $\theta$ \\
\hline McKeon et al. (2005) & EXP & $1800-32900$ & $\square$ \\
\hline Hultmark et al. (2012) & EXP & 2000-20000 & 曰 \\
\hline Furuichi et al. (2015), Furuichi et al. (2018) & EXP & $200-53000$ & $\nabla$ \\
\hline Schultz \& Flack (2013) & EXP (channel) & $1000-6000$ & 0 \\
\hline Lee \& Moser (2015) & DNS (channel) & $180-5200$ & 0 \\
\hline
\end{tabular}

Table 3. List of other references for data used in the paper.

If this trend is extrapolated, deviations of approximately $4 \%$ from the standard Prandtl law would result at $\operatorname{Re}_{b}=10^{7}$.

The mean velocity profile in turbulent pipes has received extensive attention from theoretical studies, much of the early debate being focused on whether a log law or a power law better fits the experimental results (Barenblatt, Chorin \& Prostokishin 1997), mainly carried out in the SuperPipe facility (Zagarola \& Smits 1998; McKeon et al. 2005). Recent studies have highlighted the need for corrections to the baseline log law in order to accurately describe the velocity profile throughout the log layer into the core part of the flow (Luchini 2017; Cantwell 2019; Monkewitz 2021). In figure 3, we show the series of velocity profiles computed with the present DNS, compared with previous DNS and experimental data. Overall, good agreement is observed across various sources as far as the inner and the overlap regions are concerned, with data gradually approaching a logarithmic distribution, here identified by visual fitting as $U^{+}=1 / k \log y^{+}+4.53$, using the value of $k=0.387$ determined from friction data. This is quite close to estimates 


\section{S. Pirozzoli and others}
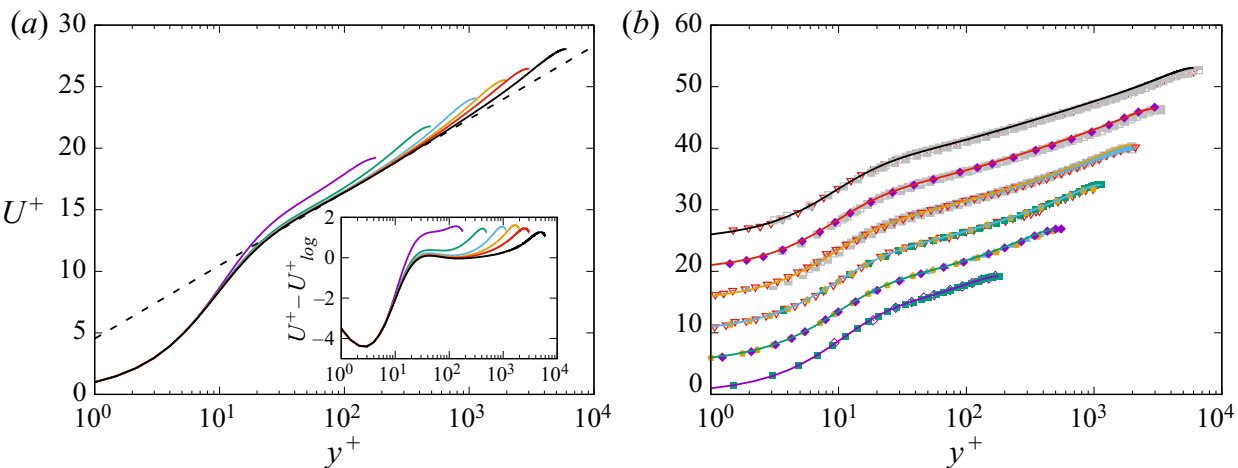

Figure 3. Inner-scaled mean velocity profiles obtained with our DNS $(a)$, and compared with previous DNS and experiments $(b)$. Deviations from the assumed logarithmic wall law, $U_{\log }^{+}=\log y^{+} / 0.387+4.53$, are highlighted in the inset of panel $(a)$. For greater clarity, profiles in panel $(b)$ are offset in the vertical direction by five wall units steps. Lines denote present DNS data, with colour code as in table 1, and symbols denote data from other authors, as in table 3.

based on direct fitting of the mean velocity profile in pipe flow (Marusic et al. 2013), which yielded $U^{+}=1 / 0.391 \log y^{+}+4.34$. The DNS velocity profiles for $\operatorname{Re}_{\tau} \geq 10^{3}$ follow this distribution with deviations of no more than 0.1 wall units from $y^{+} \approx 30$ to $y / R \approx 0.15$, whence the core region develops. Differences with respect to previous DNSs are concentrated in the core region, which seemingly stronger wake in some datasets, including our own, Wu \& Moin (2008) and Ahn et al. (2013), and weaker in others (El Khoury et al. 2013; Chin et al. 2014), reflecting previously noted differences in the friction coefficient. Especially satisfactory is the excellent agreement between our DNS-E velocity profile and the data of Ahn et al. (2015) at $R e_{\tau} \approx 3000$. Comparison of our DNS dataset with experimental data also shows overall good agreement, although some differences are quite clear in the core region, in which SuperPipe experiments consistently yield lower $U^{+}$, which translates into lower friction.

More refined information on the behaviour of the mean velocity profile can be gained from inspection of the log-law diagnostic function

$$
\Xi=y^{+} \mathrm{d} U^{+} / \mathrm{d} y^{+},
$$

which is shown in figure 4, and whose constancy would imply the presence of a genuine logarithmic layer in the mean velocity profile. The figure supports universality of the inner-scaled axial velocity for $\operatorname{Re}_{\tau} \gtrsim 10^{3}$, up to $y^{+} \approx 100$, where $\Xi$ attains a minimum, and the presence of an outer maximum at $y / R \approx 0.6$. Between these two sites the distribution is roughly linear, as can be better appreciated in figure $4(b)$, with nearly constant slope when expressed in outer coordinates. Approximate linear variation of the diagnostic function in channel flow was observed by Jiménez \& Moser (2007), who, based on refined overlap arguments expressed by Afzal \& Yajnik (1973), proposed the following fit:

$$
\Xi=\frac{1}{k}+\frac{\beta}{R e_{\tau}}+\alpha \frac{y}{R}
$$

where $\alpha, \beta$ are adjustable constants, and $k$ is the von Kármán constant. Here we find that the set of constants $k=0.387, \alpha=2.0, \beta=0$, yields overall good approximation of the pipe DNS data. The consequence is that a genuine logarithmic layer would only be attained at infinite Reynolds number. In this respect, SuperPipe data seem to suggest the formation 

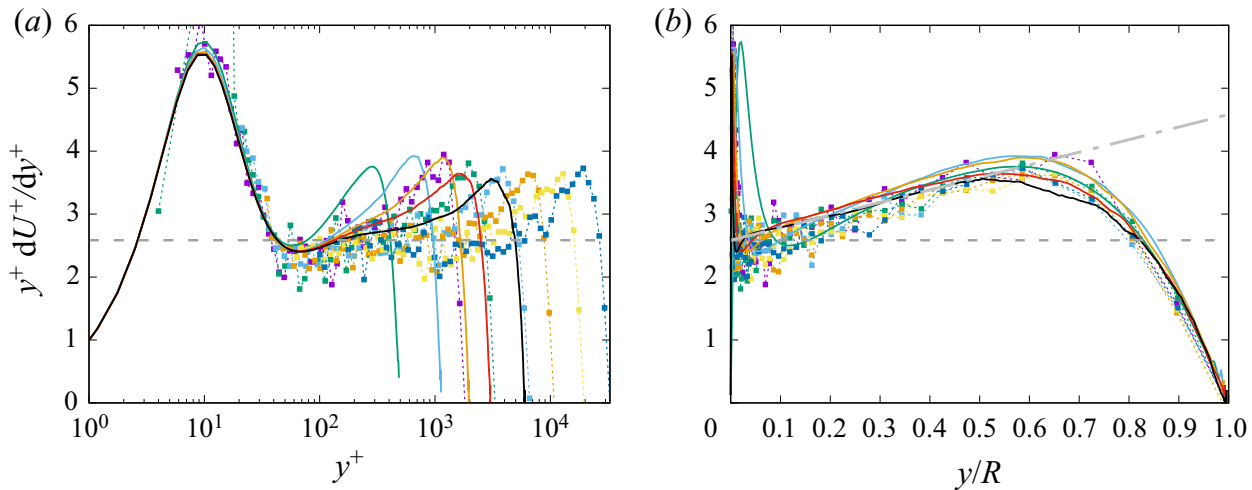

Figure 4. Log-law diagnostic function as defined in (3.3), expressed as a function of inner-scaled (a) and outer-scaled $(b)$ wall distance. The dashed horizontal line denotes the inverse Kármán constant, 1/0.387, and the dash-dotted lines in panel $(b)$ denote the linear fit (3.4), with $k=0.387, \alpha=2.0, \beta=0$. Lines denote present DNS data, with colour code as in table 1, and symbols denote SuperPipe data (McKeon et al. 2005) at $R e_{\tau}=1825,3328,6617,10914,19119,32870$.
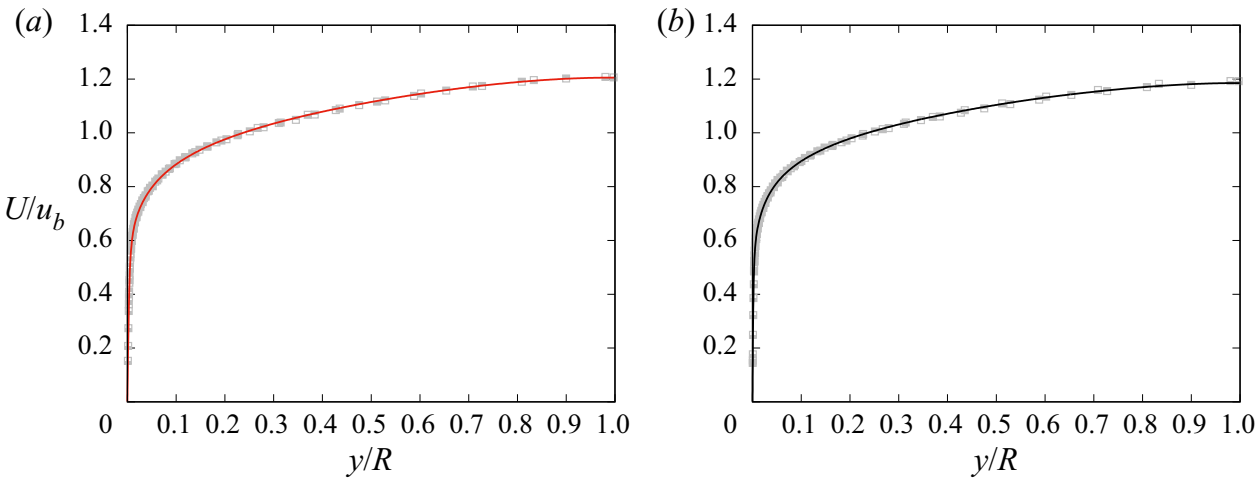

Figure 5. Mean velocity profiles in outer scaling. Data of flow case DNS-E $(a)$ are compared with SuperPipe data at $R e_{\tau}=3328$ and $R e_{\tau}=3334$, and data of flow case DNS-F (b) with SuperPipe data at $R e_{\tau}=5411$ and $R e_{\tau}=6617$.

of a plateau at $R e_{\tau} \gtrsim 10^{4}$, although the scatter of points is quite significant. Hence, DNS at higher Reynolds number would be most welcome to confirm or refute our findings, and possibly determine more accurate values of the extended log-law constants in (3.4).

Comparison with SuperPipe data is presented in outer units in figure 5, limited to the higher $R e_{\tau}$ cases. Despite differences in the Reynolds number, the velocity profiles now agree very well, throughout the outer layer. This observation would suggest problems with correct estimation of the friction velocity which, however, seems unlikely both in DNS, in which we independently evaluate friction velocity by computing the wall derivative of the velocity profile and from momentum balance, and in experiments, as measurements of the pressure drop are regarded to have low uncertainty. Hence, reasons for this discrepancy are not known, and additional experiments as those currently carried out in the large CICLoPE facility would be especially useful and welcome. Unfortunately, velocity profiles along the full radial span are not available at the moment for that facility.

The structure of the core region is examined in detail in figure 6 , where the mean velocity profiles are shown in defect form. Although full outer-layer similarity is not 


\section{S. Pirozzoli and others}
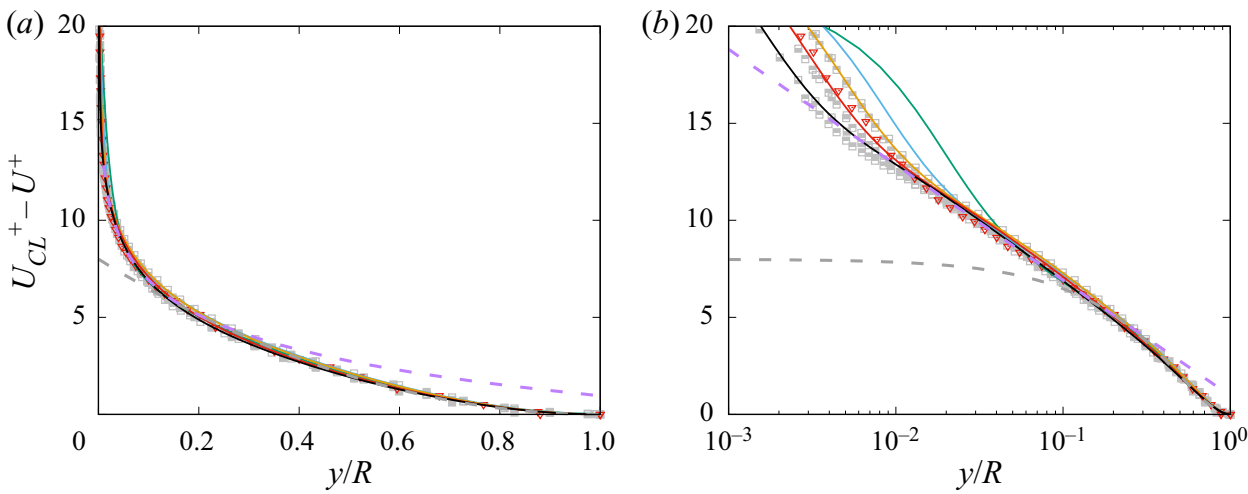

Figure 6. Defect velocity profiles for DNS and experiments, in linear $(a)$ and semilogarithmic $(b)$ scale. The dashed grey line marks a parabolic fit of the DNS data $\left(U_{C L}^{+}-U^{+}=8.0(1-y / R)^{2}\right)$, and the dashed purple line the outer-layer logarithmic fit $U_{C L}^{+}-U^{+}=0.961-1 / 0.387 \log (y / R)$.

reached at the conditions of our DNS study (also see the inset of figure $3 a$ ), scatter across the Reynolds number range and with respect to SuperPipe profiles for $y / R \geq 0.2$ is no larger than $5 \%$. As suggested by Pirozzoli (2014), the core velocity profiles can be closely approximated with a simple quadratic function, reflecting near constancy of the eddy viscosity. In particular, we find that the formula

$$
U_{C L}^{+}-U^{+}=C_{O}(1-y / R)^{2},
$$

fits the DNS data with $C_{O}=8.0$ well, and it smoothly connects at $y / R \approx 0.2$ with the logarithmic profile expressed in outer form,

$$
U_{C L}^{+}-U^{+}=-\frac{1}{k} \log (y / R)+B
$$

where again $k=0.387$, and data fitting yields $B=0.961$. While, of course, better descriptions of the core velocity profiles are possible based on more elaborate functional relationships (Luchini 2017), the composite profile matching equations (3.5) and (3.6) yields a reasonable representation of the whole outer-layer mean velocity profile within the scatter of available data.

Finer evaluation of similarities and differences between DNS and experiments is provided in figure 7, where we show the mean centreline velocity, $U_{C L}$, normalized by the friction velocity (figure $7 a$ ), and by the bulk velocity (figure $7 b$ ), as a function of the friction Reynolds number. Consistently with theoretical expectations (e.g. Monkewitz 2021), data suggest logarithmic increase with $R e_{\tau}$ according to

$$
U_{C L}^{+}=\frac{1}{k_{C L}} \log R e_{\tau}+B_{C L},
$$

where we find $k_{C L}=k=0.387$ as for the friction law, and $B_{C L}=5.85$. For convenience, the trend of $u_{b} / u_{\tau}$ is also presented, having in fact the same logarithmic growth with $R e_{\tau}$. With some previously noted differences, all pipe flow DNSs seem to exhibit a consistent trend in the accessible range. While the trend is very similar at low Reynolds number, experimental data yield consistently lower values of $U_{C L}^{+}$, especially those from the SuperPipe. At Reynolds numbers higher than approximately $R e_{\tau}=10^{4}$, experiments seem to suggest milder growth rate, although significant differences emerge between the 

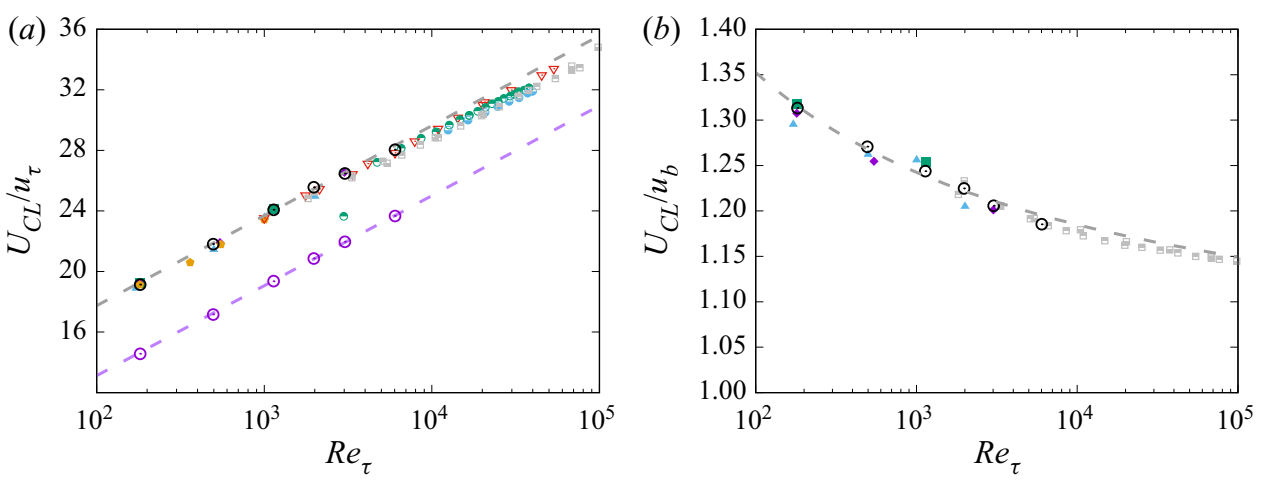

Figure 7. Mean pipe centreline velocity $\left(U_{C L}\right)$ expressed in inner $(a)$ and in outer $(b)$ units. The dashed grey line corresponds to a fit of the DNS data. The DNS data are shown as circle symbols, and the corresponding logarithmic fits are shown as thick dashed lines. Purple lines and symbols are used for the bulk velocity, $u_{b}$. For the nomenclature of other symbols, refer to table 3.

SuperPipe and the Hi-Reff datasets. Hence, whether this is the result of a change of behaviour at high Reynolds number, or some form of shortcoming of experiments, is difficult to say. As a result of the observed identity (or very close vicinity) of the von Kármán constant for the centreline and for the bulk velocity, figure 7(b) highlights that their ratio approaches unity at large $R e$, supporting the inference that pipe flow asymptotes to plug flow in the infinite-Reynolds-number limit (Pullin, Inoue \& Saito 2013). Regarding that study, it is worthwhile noticing that one of the assumptions made in the analysis is that the wall-normal location of the onset of the logarithmic region is either finite, or increases no faster than $R e_{\tau}$. Interpreting the near-wall minimum of the diagnostic function in figure 4 as the root of the (near) logarithmic layer, our data support that assumption well. Whereas the curvature of the core velocity profile is not changing substantially when expressed in wall units (see figure 6), it would become vanishingly small when expressed in outer units. However, as figure $7(b)$ suggests, this trend is extremely slow. Interestingly, again despite some scatter, DNS and experiments here seem to indicate a common trend with overall monotonic decrease, perhaps with a 'bump' in the range of Reynolds numbers in the low thousands. The DNS data points at the highest Reynolds numbers (DNS-D, DNS-E, DNS-F) now appear to be in good agreement with SuperPipe experiments, which is in line with the previously noted agreement of the outer-scaled mean velocity profiles.

The distributions of the velocity variances along the coordinate directions are shown in figure 8, in inner scaling. As is now well established (Marusic \& Monty 2019), the longitudinal $\left(u_{z}\right)$ and spanwise $\left(u_{\theta}\right)$ velocity fluctuations show slow increase with the Reynolds number, with commonly accepted logarithmic growth as after Townsend's attached eddy model (Townsend 1976). On the other hand, the wall-normal velocity fluctuations seem to level off to a maximum value of approximately 1.30. It is remarkable that the general growth of the longitudinal and spanwise fluctuations is more evident in the outer layer, and in fact it has long been argued about the possible occurrence of a secondary peak of $\left\langle u_{z}^{2}\right\rangle$, besides the primary buffer-layer peak. Experiments carried out in the SuperPipe (Hultmark et al. 2012) and CICLoPE (Willert et al. 2017) facilities indeed support the occurrence of such a peak at $R e_{\tau} \gtrsim 10^{4}$. Whereas DNS data are not at sufficiently high $R e_{\tau}$ to show this secondary peak, it appears that in DNS-F the axial velocity variance has attained a nearly horizontal inflectional point at $y^{+} \approx 140$. Comparison with the $R e_{\tau} \approx 3000$ DNS of Ahn et al. (2015) shows overall good agreement 


\section{S. Pirozzoli and others}
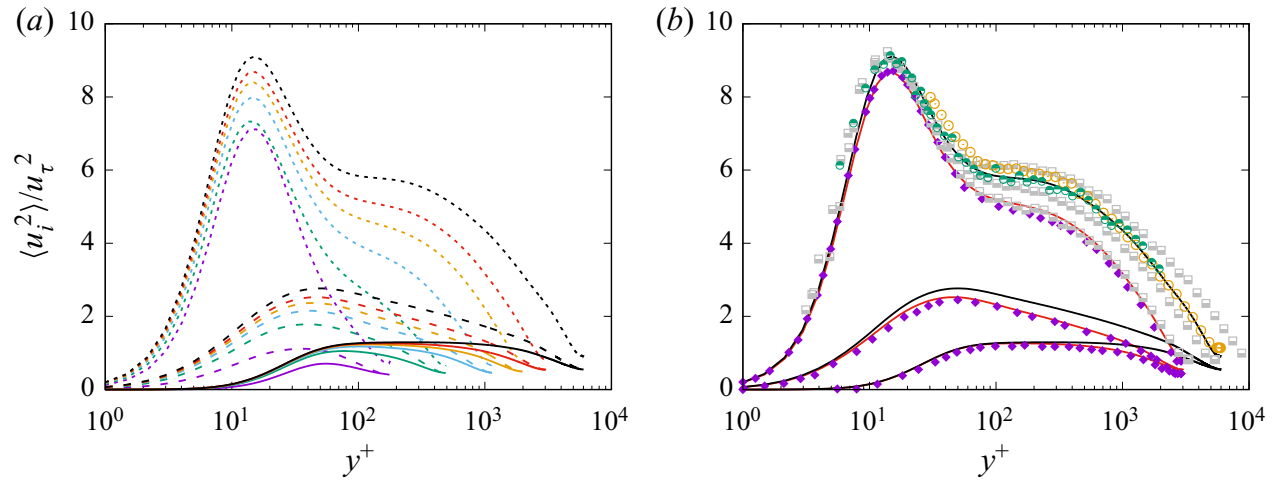

Figure 8. Distribution of velocity variances $(a)$ and comparison of cases DNS-E, DNS-F with reference DNS and experiments $(b)$. In panel $(a)$, the short dashed lines denote the axial velocity variance $\left(\left\langle u_{z}^{2}\right\rangle\right)$, the solid lines denote the radial velocity variance $\left(\left\langle u_{r}^{2}\right\rangle\right)$, and the long dashed lines denote the azimuthal velocity variance $\left(\left\langle u_{\theta}^{2}\right\rangle\right)$. For colour codes in DNS data, see table 1, and for nomenclature of symbols, see table 3.

of all turbulence intensities. Comparison with SuperPipe data at $R e_{\tau}=3000$ is also very good, with the exception of the near-wall peak which is likely to be overestimated in experiments. The DNS-F data seem to be well bracketed by SuperPipe and CICLoPE measurements at nearby Reynolds numbers, and also compare very well with experimental data for plane channel flow (Schultz \& Flack 2013).

Distributions of the turbulent shear stress are shown in figure 9. As is well established (e.g. Lee \& Moser 2015), the shear stress profiles tend to become flatter at higher $R e_{\tau}$, the peak value rises towards unity, and its position moves farther from the wall, in inner units. In particular, exploiting mean momentum balance and assuming the presence of a logarithmic layer in the mean axial velocity, the following prediction follows for the position of the turbulent shear stress peak (Afzal 1982):

$$
y_{m}^{+} \simeq \sqrt{\frac{R e_{\tau}}{k}},
$$

which is intermediate between inner and outer scaling. This observation has led some authors to argue about the relevance of a 'mesolayer' (e.g. Long \& Chen 1981; Wei et al. 2005). The asymptotic relationship (3.8) (with $k=0.387$ ) is satisfied with good accuracy starting at $R e_{\tau} \approx 10^{3}$, reflecting the onset of a near logarithmic layer. Similar results were obtained by Chin et al. (2014), by processing the mean velocity profiles obtained in the experiments of Hultmark et al. (2013).

The behaviour of the Reynolds stresses when expressed as a function of the outer-scaled wall distance, which is shown in figure 10, is also of great theoretical interest. In fact, according to the attached-eddy model (Townsend 1976; Marusic \& Monty 2019), the wall-parallel velocity variances are expected to decline logarithmically with the wall distance in the outer layer, hence

$$
\left\langle u_{z}^{2}\right\rangle=B_{1}-A_{1} \log (y / R), \quad\left\langle u_{\theta}^{2}\right\rangle=B_{3}-A_{3} \log (y / R),
$$

where $A_{i}, B_{i}$ are universal constants. Regarding the axial stress, Marusic et al. (2013) argued that SuperPipe data at the highest available Reynolds number are best fit with $A_{1}=1.23, B_{1}=1.56$, with a sensible logarithmic layer only emerging at $\operatorname{Re}_{\tau}>10^{4}$, 

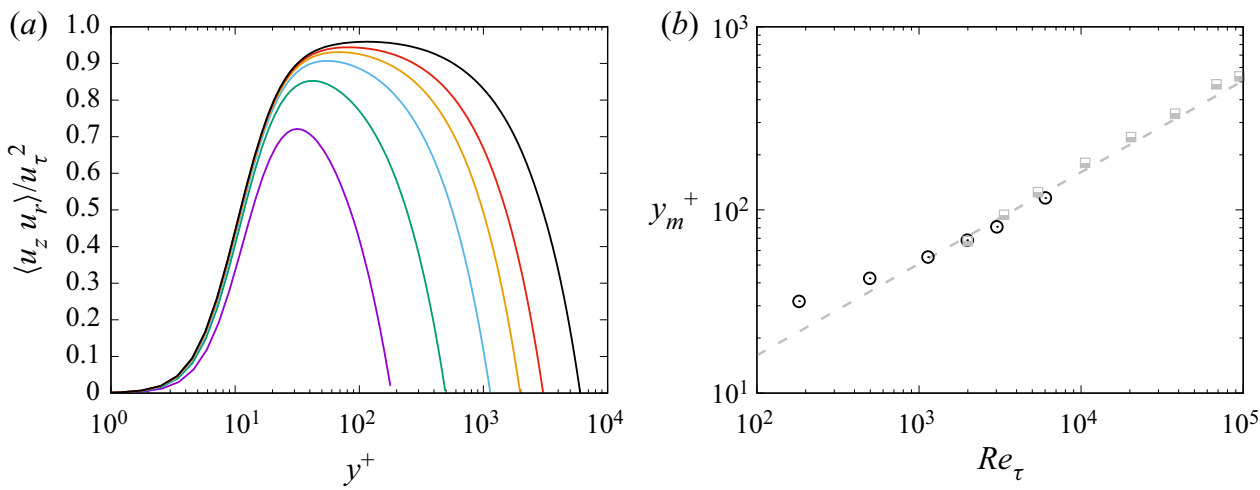

Figure 9. Distributions of turbulent shear stress $(a)$ and its peak position at various $\operatorname{Re}_{\tau}(b)$. In panel $(b)$ the circles denote the present DNS data, the squares the data of Hultmark et al. (2013), as processed by Chin et al. (2014), and the dashed line the theoretical estimate (3.8). For colour codes in DNS data, see table 1.
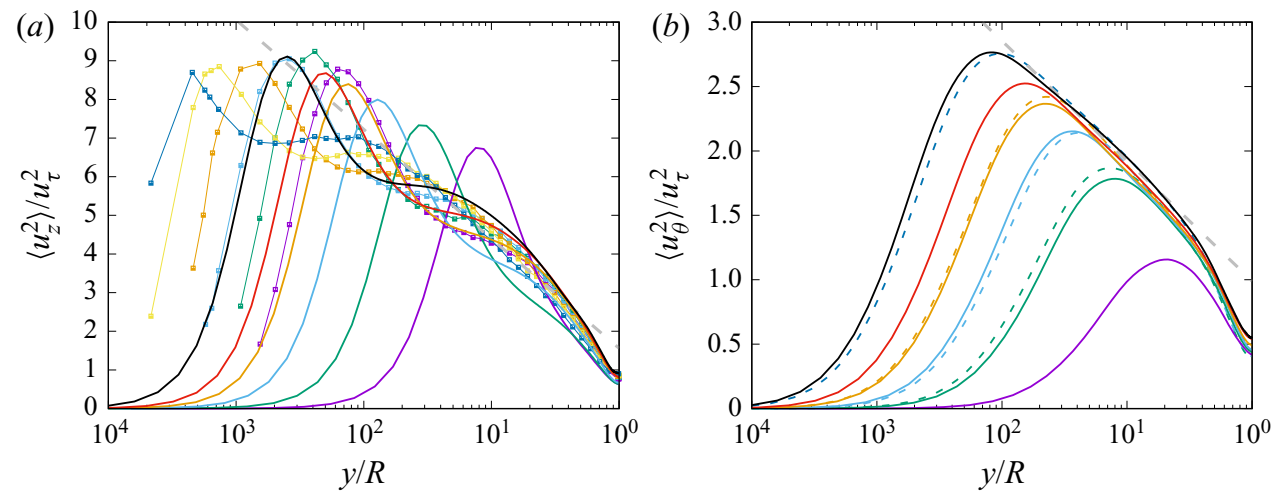

Figure 10. Axial $(a)$ and azimuthal $(b)$ turbulent stresses as a function of outer-scaled wall distance. In panel (a), symbols denote SuperPipe data (Hultmark et al. 2012) at $R e_{\tau}=1985,3334,5411,10480,20250,37690$, and the dashed grey line the corresponding fit, $\left\langle u_{z}^{2}\right\rangle=1.61-1.25 \log (y / R)$. In panel $(b)$, the dashed coloured lines denote DNS data of channel flow (Lee \& Moser 2015) at $R e_{\tau}=550,1000,2000,5200$, and the dashed grey line the fit of the DNS data, $\left\langle u_{\theta}^{2}\right\rangle=1.0-0.40 \log (y / R)$. For colour codes in DNS data, see table 1 .

in the range of wall distances $3 R e_{\tau}^{1 / 2} \leq y^{+} \leq 0.15 R e_{\tau}$. The DNS data only show the formation of a near logarithmic layer farther away from the wall, which is not where it is expected from theoretical arguments. Hence, little can be said in this respect. The azimuthal velocity variance, shown in figure $10(b)$, has a more benign behaviour, and it features clear logarithmic layers even at modest $R e_{\tau}$. Fitting the DNS data yields $A_{3}=$ $0.40, B_{3}=1.0$, which is very close to what is found in channels (Bernardini, Pirozzoli \& Orlandi 2014; Lee \& Moser 2015). Measurements of pipe flow carried out in the CICLoPE facility (Örlü et al. 2017) yielded $A_{3}=0.63, B_{3}=1.21$, hence much larger values than in DNS. Possible overestimation of the wall-normal and azimuthal Reynolds stresses was in fact acknowledged by the authors of that paper.

Quantitative insight into Reynolds number effects is provided by inspection of the amplitude of the inner peak of the axial velocity variance, which we show in figure 11 . The general theoretical expectation is that the peak grows logarithmically with $R e_{\tau}$ owing to the increasing influence of distant, inactive eddies (Marusic \& Monty 2019). However, some 


\section{S. Pirozzoli and others}
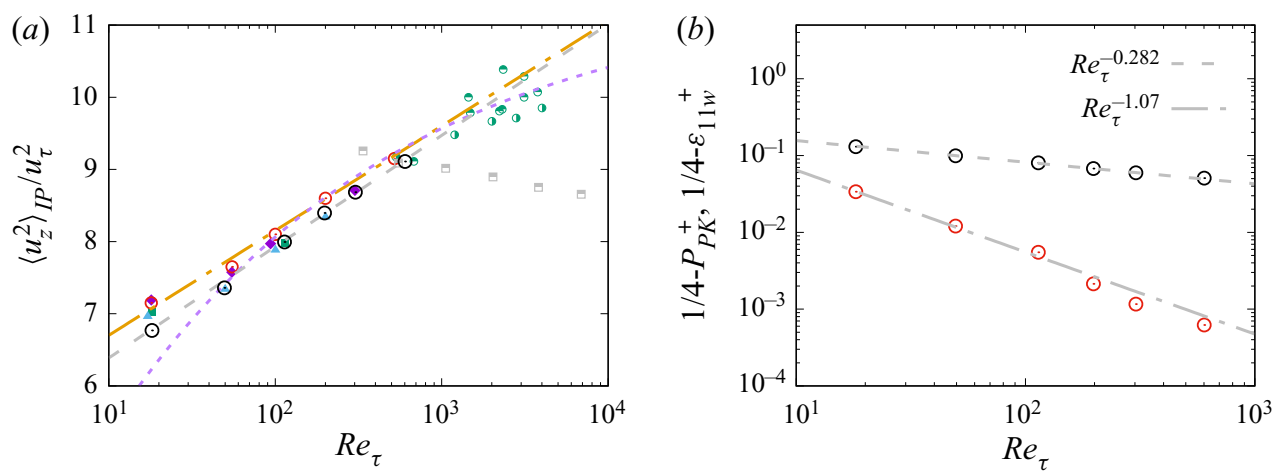

Figure 11. Magnitude of inner peak of axial velocity variance $(a)$, peak turbulence production $\left(P_{P K}\right.$, red) and wall dissipation of axial velocity variance $\left(\epsilon_{11 w}\right.$, black) $(b)$. For colour codes in DNS data, see table 1 , and for nomenclature of symbols, see table 3. In panel $(a)$ the dashed grey line marks the DNS data fit, $\left\langle u_{z}^{2}\right\rangle_{I P}^{+}=$ $0.67 \log R e_{\tau}+3.3$, the dashed purple line denotes the defect power law of Chen \& Sreenivasan (2021) and the dash-dotted line the logarithmic law of Marusic, Baars \& Hutchins (2017), $\left\langle u_{z}^{2}\right\rangle_{I P}^{+}=0.63 \log R e_{\tau}+3.8$ In panel $(b)$, the dot-dashed and dotted lines denote fits of $P_{P K}$ and $\epsilon_{11 w}$ in their tendency to the respective assumed asymptotic values.

recent experimental results (Willert et al. 2017), and theoretical arguments (Chen \& Sreenivasan 2021), suggest that such growth should eventually saturate. Although the difference between slow logarithmic growth and the attainment of an asymptotic value is quite subtle in practice, the theoretical interest is high, as in the latter case universality of wall scaling would be eventually restored. Within the investigated range of Reynolds numbers, our DNS data in fact support continuing logarithmic increase. Comparison with channel data (Lee \& Moser 2015) shows some difference, which might result from stronger geometrical confinement of distant eddies in the pipe geometry. However, differences tend to becomes smaller at higher $R_{\tau}$. In quantitative terms, we find the slope of logarithmic increase to be approximately 0.67, a bit steeper than found in channel flow DNS (Lee \& Moser 2015, approximately 0.64), and then suggested from a collection of DNS and experiments (approximately 0.63 (Marusic et al. 2017)). Experimental data for pipe flow are quite scattered, as SuperPipe experiments yield an unrealistically decreasing trend (Hultmark et al. 2012), particle image velocimetry (known as PIV) measurements taken in the CIPLoPE facility (Willert et al. 2017) suggest saturation of the growth, whereas hot-wire measurements in the same facility support continued logarithmic growth (Fiorini 2017). The theoretical predictions of Chen \& Sreenivasan (2021) (see the dashed purple line of figure 11a) seem to conform well with channel flow DNS data and with the experiments of Willert et al. (2017).

While our DNS data cannot be used to directly evaluate the theoretical predictions owing to limited achievable Reynolds number, they can be used to better scrutinize the foundations of the theoretical arguments. The main argument made by Chen \& Sreenivasan (2021), although not thoroughly justified in our opinion, was that since turbulence kinetic energy production is bounded, the wall dissipation must also stay bounded. Hence, let $P=-\left\langle u_{z} u_{r}\right\rangle \mathrm{d} U / \mathrm{d} r$ be the turbulence kinetic energy production rate, and $\epsilon_{11}=v\left\langle\left|\nabla u_{z}\right|^{2}\right\rangle$ be the dissipation rate of the axial velocity variance, those authors first argue that the wall limiting value of $\epsilon_{11}$ should scale as

$$
\epsilon_{11_{w}^{+}}^{+}=1 / 4-\beta / \operatorname{Re}_{\tau}^{1 / 4}
$$


with $\beta$ a suitable constant. In figure 11 we explore deviations of $\epsilon_{w}$ and of the peak turbulence kinetic energy production, say $P_{P K}$, from their asymptotic value, namely $1 / 4$. According to analytical constraints (Pope 2000), we find that production tends to its asymptotic value quite rapidly, as approximately $1 / R e_{\tau}$. Consistent with (3.10), the wall dissipation also tends to $1 / 4$, more or less at the predicted rate, thus empirically validating the first assumption. The next argument advocated by Chen \& Sreenivasan (2021) is that wall balance between viscous diffusion and dissipation and Taylor series expansion of the axial velocity variance near the wall yields

$$
\left\langle u_{z}^{2}\right\rangle^{+} \sim \epsilon_{11 w}^{+} y^{+^{2}}
$$

whence, from assumed invariance of the peak location of $\left\langle u_{z}^{2}\right\rangle$ (say, $y_{I P}^{+}$), saturation of growth of the peak velocity variance would follow. Table 2 suggests that this second assumption is in fact violated, as the position of the peak slightly increases with $R e_{\tau}$, with non-negligible effect on the peak variance as it appears in squared form in (3.11). As a consequence, logarithmic growth of the peak velocity variance still holds, at least in the range of Reynolds numbers currently accessible to DNS.

A secondary, outer-layer peak of the axial velocity variance was observed in the SuperPipe experiments of Hultmark et al. (2012), which relied on nanoscale thermal anemometry probes. Later experiments carried out in the CICLoPE facility (Örlü et al. 2017), using custom-made X-wire probes, raised doubts about the existence of a genuine outer peak, and in general prompted further high-quality data to ascertain whether it exists beyond measurement uncertainty. Particle image velocimetry measurements also carried out in the CICLoPE facility (Willert et al. 2017), did show an outer peak that develops and moves away from the inner peak with increasing Reynolds number. Hence, it is clear that this issue is not definitely settled in experiments. Although no distinct outer peak of the axial velocity variance is found at the Reynolds numbers accessed in the present DNS study, it is nevertheless instructive to explore the scaling of the velocity fluctuations in the range of wall distances where the peak is expected to occur. For that purpose, we consider the outer position where the second logarithmic derivative of the velocity variance vanishes, which in the present DNS ranges from $y^{+} \approx 115$ for DNS-A, to $y^{+} \approx 140$ for DNS-F. Weak dependence of the inner-scaled outer peak position on $R e_{\tau}$, although at much higher Reynolds number, was also noticed by Hultmark et al. (2012). The resulting distribution is shown in figure 12 . All DNS data fall nicely on a logarithmic fit, and they seem to connect smoothly to the experimental results, whose scatter and uncertainty is expected to be much less than for the inner peak. Experiments indicate a change of behaviour to a shallower logarithmic dependence with slope of approximately 0.63 (Pullin et al. 2013; Fiorini 2017), which would be very close to the growth rate of the inner peak (see figure 11). The figure suggests that verification of this effect would require $R e_{\tau}$ of approximately $10^{4}$.

As pointed out by Hultmark et al. (2012), the formation and growth of an outer peak of the axial velocity variance has important theoretical and practical implications. From the modelling standpoint, no current Reynolds-averaged Navier-Stokes (RANS) model is capable of predicting non-monotonic behaviour of Reynolds stresses outside the buffer layer. From the fundamental physics standpoint, the presence of an outer peak is suggestive of violation of equilibrium between turbulence production and dissipation. The DNS allows us to substantiate this scenario, and for that purpose in figure 13, we show the relative excess of turbulent kinetic energy production $(P)$ over its total dissipation rate, here defined as $D=v\left\langle u_{i} \nabla^{2} u_{i}\right\rangle$, which lumps together dissipation rate and viscous diffusion. Data confirm the presence of a near-universal region confined to the buffer layer (say, 


\section{S. Pirozzoli and others}

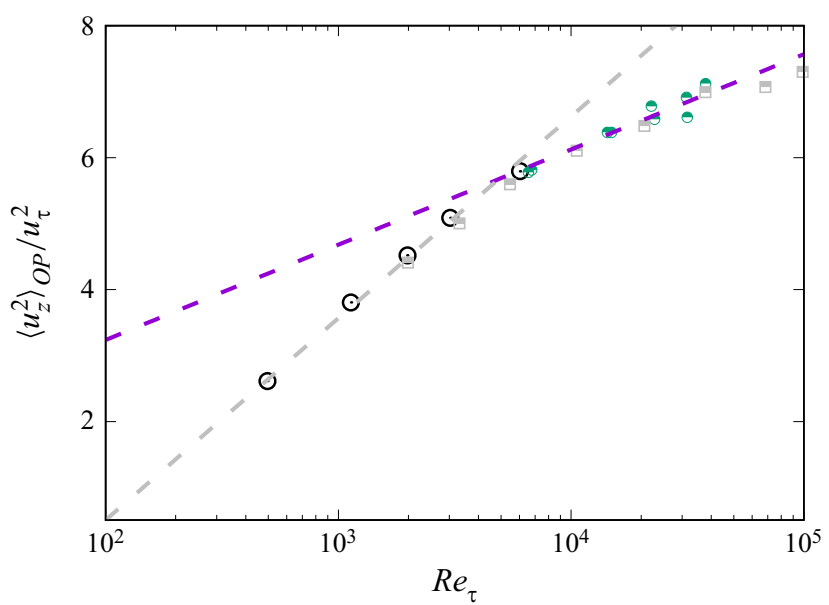

Figure 12. Magnitude of outer peak of axial velocity variance as a function of $R e_{\tau}$. Lines and symbols as in tables 1 and 3 . The dashed grey line marks the DNS data fit, $\left\langle u_{z}^{2}\right\rangle_{O P}^{+}=1.33 \log R e_{\tau}-5.61$, and the purple line denotes the logarithmic fit given by Pullin et al. (2013).
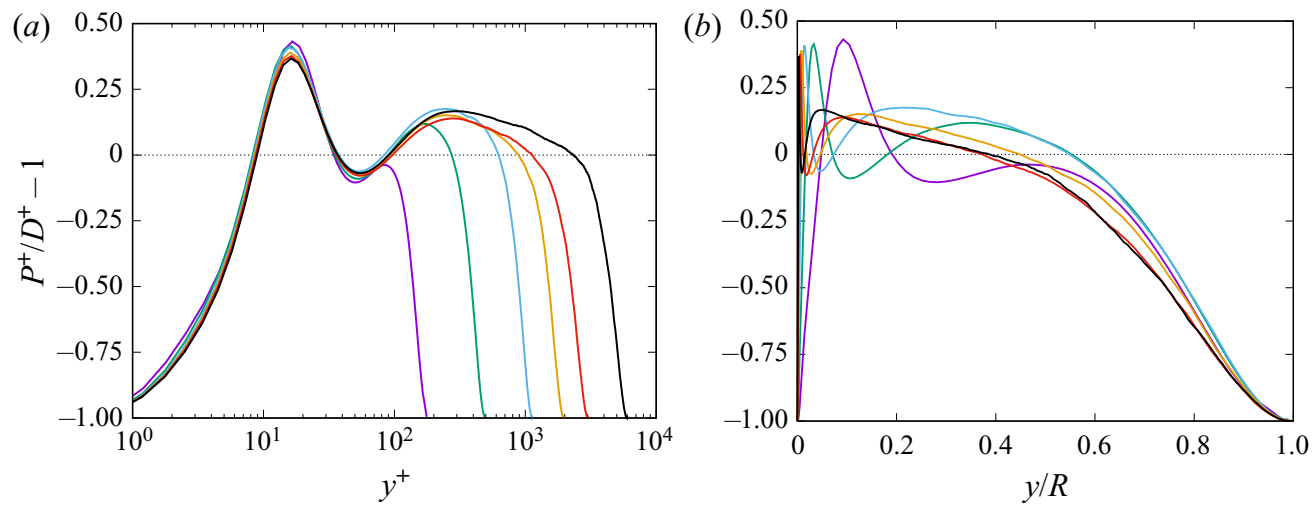

Figure 13. Excess of turbulence kinetic energy production over dissipation as a function of inner-scaled $(a)$ and outer-scaled $(b)$ wall distance. Lines as in table 1.

$8 \lesssim y^{+} \lesssim 35$ ), in which production exceeds dissipation by up to $40 \%$. Data also show the onset, starting from DNS-B, of another region farther from the wall with positive unbalance, whose inner limit is constant in inner units, at $y^{+}=100$, and whose outer limit tends to become constant at high $R e_{\tau}$ in outer units, at $y / R \approx 0.4$. The peak unbalance at high Reynolds number is approximately $17 \%$, and its position seems to scale more in inner than in outer units. Turbulence kinetic energy production excess in the presence of a (near) logarithmic mean velocity profile can be interpreted by recalling that only part of the axial velocity fluctuations which are generated correlates with wall-normal velocity fluctuations to yield active motions (Townsend 1976), hence the extra production feeds inactive motions, which do not convey contribution to the turbulent shear stress. This finding clearly indicates that at high enough Reynolds number the outer wall layer becomes a dynamically active part of the flow, having the potential to transfer energy both to the core flow, and towards the wall, in the form of imprinting on the near-wall layer (Marusic \& Monty 2019). 


\section{Concluding comments}

Although DNS of wall turbulence is still confined to a moderate range of Reynolds numbers, it is beginning to approach a state in which some typical phenomena of the asymptotically high-Re emerge. Given its ability to resolve the near-wall layer, DNS lends itself to testing theories of wall turbulence and to in-depth scrutiny of experimental data. In this work, DNS of flow in a smooth pipe has been carried out up to $\operatorname{Re}_{\tau} \approx 6000$, which, although still far from what achievable in experimental tests, allows us to uncover a number of interesting issues, in our opinion. First, we have noted that DNS data fall systematically short of the classical Prandtl friction law, by as much as $2 \%$. This evidence is not consistent with data from the SuperPipe facility, although other recent data from the CICLoPE and Hi-Reff facilities seem to yield similar trends. The DNS data fitting suggests that a logarithmic law as (3.2) still holds, however, with a von Kármán constant $k \approx 0.387$, which matches extremely well the value quoted by Furuichi et al. (2018), and which would reconcile pipe flow with plane channel and boundary layer flows, thus corroborating claims made by Marusic et al. (2013). A logarithmic profile with $k \approx 0.387$ fits the mean axial velocity distributions for $30 \leq y^{+} \leq 0.15 R e_{\tau}$ well, although linear deviations are clearly visible, as argued by Afzal \& Yajnik (1973) and Luchini (2017), which when taken into account yield an excellent representation of the velocity profiles up to $y / R \approx 0.5$. It is remarkable that the same value of the von Kármán constant also fits the mean centreline velocity distribution well (see figure 7), which is found to grow logarithmically throughout the range of $R e_{\tau}$ under investigation. This finding is quite reasonable as it corroborates that the eventual state of turbulent flow in a pipe should be plug flow, as argued by Pullin et al. (2013), hence $U_{C L} \rightarrow u_{b}$ as $R e_{\tau} \rightarrow \infty$. This would, however, seemingly contrast with recent measurements made in the CICLoPE facility (Nagib et al. 2017), which rather suggest a different von Kármán constant for the bulk and the centreline velocity. Experimental data at $R e_{\tau} \gtrsim 10^{4}$ in fact suggest deviations of $U_{C L}^{+}$from the logarithmic trend found in DNS, however, this effect requires further confirmation, as data are quite scattered. The core velocity profile is found to be, to a good approximation, parabolic, with curvature which is nearly constant in wall units, and decreasing in outer units.

Regarding the velocity fluctuations, we find evidence for continuing logarithmic increase of the inner-peak magnitude with $R e_{\tau}$. Some experiments and theoretical arguments would indicate that beyond $R e_{\tau} \approx 10^{4}$ a change of behaviour might occur which, however, is very difficult to quantify. The DNS is probably of little use in this respect, as in order to clearly discern among the various trends, $R e_{\tau}$ in excess of $10^{5}$ are likely to be needed. As predicted by the attached-eddy hypothesis, the wall-parallel velocity variances in the outer layer tend to form logarithmic layers, which are especially evident in the azimuthal velocity. Although we do not find direct evidence for the existence of an outer peak of the axial velocity variance, our results highlight the occurrence of an outer site with substantial turbulence production excess over dissipation, thus contradicting the classical equilibrium hypothesis and likely to yield a distinct peak at $\operatorname{Re}_{\tau} \approx 10^{4}$. Investigating these and other violations of universality of wall turbulence to extrapolate asymptotic behaviours is a formidable challenge for theoreticians in years to come.

Supplementary movies. Supplementary movies are available at https://doi.org/10.1017/jfm.2021.727.

Acknowledgements. We acknowledge that the results reported in this paper have been achieved using the PRACE Research Infrastructure resource MARCONI based at CINECA, Casalecchio di Reno, Italy, under project PRACE no. 2019204979. Discussions with A.J. Smits are gratefully acknowledged. We would like to thank P. Luchini and M. Quadrio for providing the code used for the data uncertainty analysis. 


\section{S. Pirozzoli and others}

Funding. This research received no specific grant from any funding agency, commercial or not-for-profit sectors.

Declaration of interests. The authors report no conflict of interest.

Data availability statement. The data that support the findings of this study are openly available at the web page http://newton.dma.uniroma1.it/database/

\section{Author ORCIDs.}

(1) Sergio Pirozzoli https://orcid.org/0000-0002-7160-3023;

(D) Roberto Verzicco https://orcid.org/0000-0002-2690-9998;

D Paolo Orlandi https://orcid.org/0000-0002-0305-5723.

\section{REFERENCES}

AfZAL, N. 1982 Fully developed turbulent flow in a pipe: an intermediate layer. Ing. Arch. 52 (6), 355-377.

AfZAL, N. \& YAJNIK, K. 1973 Analysis of turbulent pipe and channel flows at moderately large Reynolds number. J. Fluid Mech. 61, 23-31.

Ahn, J., LeE, J.H., JANG, S. \& Sung, H.J. 2013 Direct numerical simulations of fully developed turbulent pipe flows for $R e_{\tau}=180,544$ and 934. Intl J. Heat Fluid Flow 44, 222-228.

Ahn, J., LeE, J.H., LeE, J., KAng, J.-H. \& Sung, H.J. 2015 Direct numerical simulation of a 30R long turbulent pipe flow at $R e_{\tau}=3000$. Phys. Fluids 27, 065110.

AKselvoll, K. \& Moin, P. 1996 An efficient method for temporal integration of the Navier-Stokes equations in confined axisymmetric geometries. J. Comput. Phys. 125, 454-463.

Barenblatt, G.I., Chorin, A.J. \& Prostokishin, V.M. 1997 Scaling laws for fully developed turbulent flow in pipes. Appl. Mech. Rev. 50, 413-429.

Bernardini, M., Pirozzoli, S. \& Orlandi, P. 2014 Velocity statistics in turbulent channel flow up to $R e_{\tau}=4000$. J. Fluid Mech. 742, 171-191.

CANTwell, B.J. 2019 A universal velocity profile for smooth wall pipe flow. J. Fluid Mech. 878, 834-874.

Chen, X. \& SReenivasan, K.R. 2021 Reynolds number scaling of the peak turbulence intensity in wall flows. J. Fluid Mech. 908, R3.

Chin, C., Ooi, A.S.H., Marusic, I. \& Blackburn, H.M. 2010 The influence of pipe length on turbulence statistics computed from direct numerical simulation data. Phys. Fluids 22 (11), 115107.

Chin, C., Philip, J., Klewicki, J., OOI, A. \& Marusic, I. 2014 Reynolds-number-dependent turbulent inertia and onset of log region in pipe flows. J. Fluid Mech. 757, 747-769.

DURST, F., JOVANOVIĆ, J. \& SENDER, J. 1995 LDA measurements in the near-wall region of a turbulent pipe flow. J. Fluid Mech. 295, 305-335.

Eggels, J.G.M., Unger, F., Weiss, M.H., Westerweel, J., Adrian, R.J., Friedrich, R. \& NieuwstadT, F.T.M. 1994 Fully developed turbulent pipe flow: a comparison between direct numerical simulation and experiment. J. Fluid Mech. 268, 175-210.

El Khoury, G.K., Schlatter, P., Noorani, A., Fischer, P.F., Brethouwer, G. \& Johansson, A.V. 2013 Direct numerical simulation of turbulent pipe flow at moderately high Reynolds numbers. Flow Turbul. Combust. 91, 475-495.

FIORINI, T. 2017 Turbulent pipe flow - high resolution measurements in CICLoPE. PhD thesis, School of Engineering and Architecture, University of Bologna.

Furuichi, N., Terao, Y., WADA, Y. \& TsujI, Y. 2015 Friction factor and mean velocity profile for pipe flow at high Reynolds numbers. Phys. Fluids 27, 095108.

Furuichi, N., TERAO, Y., WAdA, Y. \& Tsuji, Y. 2018 Further experiments for mean velocity profile of pipe flow at high Reynolds number. Phys. Fluids 29, 055101.

HARLOW, F.H. \& WelCh, J.E. 1965 Numerical calculation of time-dependent viscous incompressible flow of fluid with free surface. Phys. Fluids 8, 2182-2189.

Hellström, L.H.O. \& SMits, A.J. 2014 The energetic motions in turbulent pipe flow. Phys. Fluids 26, 125102.

HoyAS, S. \& JimÉnEZ, J. 2006 Scaling of velocity fluctuations in turbulent channels up to $R e_{\tau}=2003$. Phys. Fluids 18, 011702.

Hultmark, M., Bailey, S.C.C. \& Smits, A.J. 2010 Scaling of near-wall turbulence in pipe flow. J. Fluid Mech. 649, 103-113.

Hultmark, M., Vallikivi, M., Bailey, S.C.C. \& Smits, A.J. 2012 Turbulent pipe flow at extreme Reynolds numbers. Phys. Rev. Lett. 108, 094501. 
Hultmark, M., Vallikivi, M., Bailey, S.C.C. \& Smits, A.J. 2013 Logarithmic scaling of turbulence in smooth-and rough-wall pipe flow. J. Fluid Mech. 728, 376-395.

JiMÉNEZ, J. 2018 Coherent structures in wall-bounded turbulence. J. Fluid Mech. 842, P1.

JiméneZ, J. \& Moser, R.D. 2007 What are we learning from simulating wall turbulence? Phil. Trans. R. Soc. Lond. A 365, 715-732.

KiM, J. \& Moin, P. 1985 Application of a fractional-step method to incompressible Navier-Stokes equations. J. Comput. Phys. 59, 308-323.

LeE, M. \& Moser, R.D. 2015 Direct simulation of turbulent channel flow layer up to $\operatorname{Re}_{\tau}=5200$. J. Fluid Mech. 774, 395-415.

LONG, R.R. \& CHEN, T.-C. 1981 Experimental evidence for the existence of the 'mesolayer' in turbulent systems. J. Fluid Mech. 105, 19-59.

LuCHINI, P. 2017 Universality of the turbulent velocity profile. Phys. Rev. Lett. 118 (22), 224501.

Marusic, I., BAARS, W.J. \& Hutchins, N. 2017 Scaling of the streamwise turbulence intensity in the context of inner-outer interactions in wall turbulence. Phys. Rev. Fluids 2, 100502.

Marusic, I. \& Monty, J.P. 2019 Attached eddy model of wall turbulence. Annu. Rev. Fluid Mech. 51, 49-74.

Marusic, I., Monty, J.P., Hultmark, M. \& Smits, A.J. 2013 On the logarithmic region in wall turbulence. J. Fluid Mech. 716, R3.

McKeon, B.J., Zagarola, M.V. \& Smits, A.J. 2005 A new friction factor relationship for fully developed pipe flow. J. Fluid Mech. 538, 429-443.

MoIN, P. \& VERZICCO, R. 2016 On the suitability of second-order accurate discretizations for turbulent flow simulations. Eur. J. Mech. B/Fluids 55, 242-245.

Monkewitz, P.A. 2021 The late start of the mean velocity overlap log law at $y^{+}=O\left(10^{3}\right)-$ a generic feature of turbulent wall layers in ducts. J. Fluid Mech. 910, A45.

NAGIB, H.M. \& CHAUHAN, K.A. 2009 Criteria for assessing experiments in zero pressure gradient boundary layers. Fluid Dyn. Res. 41, 021404.

Nagib, H.M., Monkewitz, P.A., Mascotelli, L., Fiorini, T., Bellani, G., Zheng, X. \& Talamelli, A. 2017 Centerline Kármán 'constant' revisited and contrasted to log-layer Kármán constant at CICLoPE. In 10th International Symposium on Turbulence and Shear Flow Phenomena.

NIKURADSE, J. 1933 Stromungsgesetze in rauhen Rohren. VDI-Forschungsheft 361, 1.

ORLANDi, P. \& FATICA, M. 1997 Direct simulations of turbulent flow in a pipe rotating about its axis. J. Fluid Mech. 343, 43-72.

Örlü, R., Fiorini, T., Segalini, A., Bellani, G., Talamelli, A. \& Alfredsson, P.H. 2017 Reynolds stress scaling in pipe flow turbulence - first results from CICLoPe. Phil. Trans. R. Soc. Lond. A 375 (2089), 20160187.

PIROzZOLI, S. 2014 Revisiting the mixing-length hypothesis in the outer part of turbulent wall layers: mean flow and wall friction. J. Fluid Mech. 745, 378-397.

Pirozzoli, S., Bernardini, M. \& Orlandi, P. 2016 Passive scalars in turbulent channel flow at high Reynolds number. J. Fluid Mech. 788, 614-639.

Pirozzoli, S. \& Orlandi, P. 2021 Natural grid stretching for DNS of wall-bounded flows. J. Comput. Phys. 439, 110408.

Pope, S.B. 2000 Turbulent Flows. Cambridge University Press.

Pullin, D.I., InOUe, M. \& SAito, N. 2013 On the asymptotic state of high Reynolds number, smooth-wall turbulent flows. Phys. Fluids 25, 015116.

REYNOLDS, O. 1883 An experimental investigation of the circumstances which determine whether the motion of water shall be direct or sinuous, and of the law of resistance in parallel channels. Phil. Trans. R. Soc. Lond. 174, 935-982.

Ruetsch, G. \& FATICA, M. 2014 CUDA Fortran for Scientists and Engineers. Elsevier.

Russo, S. \& LuCHINI, P. 2017 A fast algorithm for the estimation of statistical error in DNS (or experimental) time averages. J. Comput. Phys. 347, 328-340.

Schultz, M.P. \& Flack, K.A. 2013 Reynolds-number scaling of turbulent channel flow. Phys. Fluids 25, 025104.

Stevens, R.J.A.M., van der Poel, E.P., Grossmann, S. \& Lohse, D. 2013 The unifying theory of scaling in thermal convection: the updated prefactors. J. Fluid Mech. 730, 295-308.

Swanson, C.J., Julian, B., Ihas, G.G. \& Donnelly, R.J. 2002 Pipe flow measurements over a wide range of Reynolds numbers using liquid helium and various gases. J. Fluid Mech. 461, 51-60.

Townsend, A.A. 1976 The Structure of Turbulent Shear Flow, 2nd edn. Cambridge University Press.

VERZICCO, R. \& ORLANDI, P. 1996 A finite-difference scheme for three-dimensional incompressible flows in cylindrical coordinates. J. Comput. Phys. 123, 402-414. 


\section{S. Pirozzoli and others}

Wei, T., Fife, P., Klewicki, J. \& McMurtry, P. 2005 Properties of the mean momentum balance in turbulent boundary layer, pipe and channel flow. J. Fluid Mech. 573, 303-327.

Willert, C.E., Soria, J., Stanislas, M., Klinner, J., Amili, O., Eisfelder, M., Cuvier, C., Bellani, G., Fiorini, T. \& Talamelli, A. 2017 Near-wall statistics of a turbulent pipe flow at shear Reynolds numbers up to 40 000. J. Fluid Mech. 826, R5.

Wu, X. \& MoIN, P. 2008 A direct numerical simulation study on the mean velocity characteristics in turbulent pipe flow. J. Fluid Mech. 608, 81-112.

ZaGarola, M.V. \& SMits, A.J. 1998 Mean-flow scaling of turbulent pipe flow. J. Fluid Mech. 373, 33-79. 